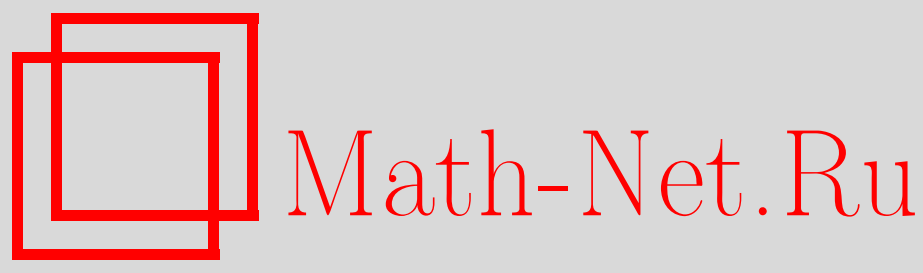

А. И. Мудров, Регуляризация генераторов Микельсона для неисключительных квантовых групп, ТМФ, 2017, том 192, номер 2, 307-321

DOI: https://doi.org/10.4213/tmf9299

Использование Общероссийского математического портала Math-Net.Ru подразумевает, что вы прочитали и согласны с пользовательским соглашением http://www . mathnet.ru/rus/agreement

Параметры загрузки:

IP: 54.198 .55 .26

26 апреля 2023 г., 16:11:39

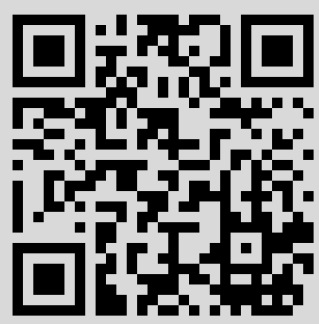




\section{РЕГУЛЯРИЗАЦИЯ ГЕНЕРАТОРОВ МИКЕЛЬСОНА ДЛЯ НЕИСКЛЮЧИТЕЛЬНЫХ КВАНТОВЫХ ГРУПП}

Пусть $\mathfrak{g}^{\prime} \subset \mathfrak{g}-$ пара алгебр Ли симплектических или ортогональных инфинитезимальных эндоморфизмов комплексных векторных пространств $\mathbb{C}^{N-2} \subset \mathbb{C}^{N}$, а $U_{q}\left(\mathfrak{g}^{\prime}\right) \subset U_{q}(\mathfrak{g})$ - пара квантовых групп с треугольным разложением $U_{q}(\mathfrak{g})=$ $U_{q}\left(\mathfrak{g}_{-}\right) U_{q}\left(\mathfrak{g}_{+}\right) U_{q}(\mathfrak{h})$. Пусть $Z_{q}\left(\mathfrak{g}, \mathfrak{g}^{\prime}\right)$ - соответствующая редукционная алгебра, генераторами которой, как мы считаем, являются рациональные тригонометрические функции $\mathfrak{h}^{*} \rightarrow U_{q}\left(\mathfrak{g}_{ \pm}\right)$. Рассмотрена такая их регуляризация, что итоговые генераторы не обращаются в нуль при любом выборе веса.

Ключевые слова: алгебры Микельсона, квантовые группы, регуляризация.

DOI: https://doi.org/10.4213/tmf9299

Посвящается памяти П. П. Кулиша

\section{1. ВВЕДЕНИЕ}

Содержание данной статьи имеет технический вид, она посвящена регуляризации генераторов алгебр Микельсона, которые рассматриваются как мероморфные функции на пространстве весов. С общей теорией алгебр Микельсона читатель может ознакомиться в работах [1]-[3] (классические универсальные обертывающие алгебры) и [4], [5] (квантовые группы). Здесь нас интересует частный случай, соответствующий паре $\mathfrak{g}^{\prime} \subset \mathfrak{g}$ алгебр Ли ортогональных или симплектических инфинитезимальных преобразований фиксированной пары векторных пространств $\mathbb{C}^{N-2} \subset \mathbb{C}^{N}$.

Пусть $\mathfrak{g}^{\prime}=\mathfrak{g}_{-}^{\prime} \oplus \mathfrak{h}^{\prime} \oplus \mathfrak{g}_{+}^{\prime}-$ треугольное разложение, совместное с разложением $\mathfrak{g}=\mathfrak{g}_{-} \oplus \mathfrak{h} \oplus \mathfrak{g}_{+}$, т. е. $\mathfrak{g}_{ \pm}^{\prime} \subset \mathfrak{g}_{ \pm}$, где $\mathfrak{h}^{\prime} \subset \mathfrak{h}-$ подалгебры Картана. Пусть $N\left(\mathfrak{g}, \mathfrak{g}^{\prime}\right)$ означает нормализатор левого идеала $U_{q}(\mathfrak{g}) \mathfrak{g}_{+}^{\prime}$, т. е. максимальную подалгебру в $U_{q}(\mathfrak{g})$, в которой $U_{q}(\mathfrak{g}) \mathfrak{g}_{+}^{\prime}$ является двусторонним идеалом. Тогда фактор $N\left(\mathfrak{g}, \mathfrak{g}^{\prime}\right) / U_{q}(\mathfrak{g}) \mathfrak{g}_{+}^{\prime}$ называется редукционной алгеброй, или алгеброй Микельсона и обозначается как $Z_{q}\left(\mathfrak{g}, \mathfrak{g}^{\prime}\right)$. Ее важность вытекает из того факта, что она сохраняет подпространство $\mathfrak{g}^{\prime}$-сингулярных векторов в $\mathfrak{g}$-модулях (напомним, что вектор называется сингулярным, если он порождает тривиальное представление алгебры $\left.U_{q}\left(\mathfrak{g}_{+}^{\prime}\right)\right)$.

Работа частично поддержана РФФИ (грант № 15-01-03148).

${ }^{*}$ Mathematics Department, University of Leicester, United Kingdom. E-mail: am405@le.ac.uk 
Алгебра Микельсона снабжена двухсторонним $U_{q}(\mathfrak{h})$-действием и порождается элементами $z_{0}, z_{ \pm \alpha}$ с весами 0 и соответственно $\pm \alpha$, где $\alpha \in \mathrm{R}_{\mathfrak{g}}^{+}-\mathrm{R}_{\mathfrak{g}^{\prime}}^{+}$(множеству положительных корней алгебры $\mathfrak{g}$ за исключением положительных корней алгебры $\left.\mathfrak{g}^{\prime}\right)$. Элемент $z_{0}$ можно взять из $q^{\mathfrak{h}^{\ominus \mathfrak{h}^{\prime}}}$, а $z_{ \pm \alpha}$ имеет представителей в подалгебре Бореля $U_{q}\left(\mathfrak{b}_{ \pm}\right)$. Генераторы $z_{ \pm \alpha}$ можно выразить через экстремальные проекторы [6]-[8] или, иначе, как элементы редуцированной обратной матрицы Шаповалова [9], [10] (классическая версия приведена в работах [11]-[13]). В обоих случаях требуется рациональное расширение $\widehat{U}_{q}(\mathfrak{g})$ группы $U_{q}(\mathfrak{g})$ над кольцом частных $\widehat{U}_{q}(\mathfrak{h})$ группы $U_{q}(\mathfrak{h})$ по отношению к некоторой мультипликативной системе.

Что касается $\widehat{U}_{q}(\mathfrak{g})$ как свободного правого $\widehat{U}_{q}(\mathfrak{h})$-модуля, то можно считать $z_{ \pm \alpha}$ рациональными тригонометрическими $U_{q}\left(\mathfrak{g}_{ \pm}\right)$-значными функциями (повышающими и понижающими операторами) на $\mathfrak{h}^{*}$. Конечно, их можно превратить в полиномы посредством умножения на общий знаменатель картановских коэффициентов. Мы будем называть этот процесс естественной регуляризацией и обозначим регуляризованные генераторы через $\check{z}_{ \pm \alpha}$. Возникает вопрос обращения в ноль элементов $\check{z}_{ \pm \alpha}(\lambda)=0$ при некотором $\lambda$. Ответ на этот вопрос дается в настоящей работе. Для специального случая общих линейных и симплектических алгебр $\mathfrak{g}$ мы доказываем, что $\check{z}_{ \pm \alpha}(\lambda) \neq 0$ при всех $\lambda \in \mathfrak{h}^{*}$. Для ортогональной алгебры $\mathfrak{g}$ существуют такие элементы $\delta_{ \pm \alpha} \in U_{q}(\mathfrak{h})$, что $\check{z}_{ \pm \alpha}$ делятся на $\delta_{ \pm \alpha}$ справа, а фактор $\check{z}_{ \pm \alpha} \delta_{ \pm \alpha}^{-1}$ не обращается в ноль для всех весов.

Задача регуляризации отрицательных генераторов Микельсона близка к задаче регуляризации сингулярных векторов группы $V \otimes M_{\lambda}$, где $V$ - "естественное" представление группы $U_{q}(\mathfrak{g})$, а $M_{\lambda}$ - модуль Верма старшего веса $\lambda$ : сингулярные векторы являются рациональными тригонометрическими функциями веса $\lambda$. Задача в такой постановке является частью более общей проблемы, в которой $V$ - произвольный конечномерный $U_{q}(\mathfrak{g})$-модуль. Аналогичная проблема рассматривалась для классических универсальных обертывающих алгебр в работе [14] и полностью решена для $\mathfrak{g}=\mathfrak{s l}(3)$. Здесь мы изучаем частный случай $V=\mathbb{C}^{N}$ в силу его важности для редукционных алгебр. Заметим, что квантовые группы приводят к новым эффектам, которые отсутствуют в классической версии: факторы $\delta_{ \pm \alpha}$ обращаются в единицу для $\mathfrak{g}=\mathfrak{s o}(2 n+1)$ в пределе $q \rightarrow 1$.

\section{2. ПРЕДВАРИТЕЛЬНЫЕ СВЕДЕНИЯ ИЗ ТЕОРИИ КВАНТОВЫХ ГРУПП}

Далее в работе символ $\mathfrak{g}$ означает комплексную простую алгебру Ли типа $B, C$ или $D$. Ввиду естественного вложения $U_{q}(\mathfrak{g l}(n)) \subset U_{q}(\mathfrak{g})$ мы не уделяем особого внимания этому случаю (который также рассмотрен в следствии 9.2 статьи [15], но с другой точки зрения). Зафиксируем подалгебру Картана $\mathfrak{h} \subset \mathfrak{g}$ с невырожденным симметричным скалярным произведением $(\cdot, \cdot)$ на $\mathfrak{h}^{*}$. Обозначим R систему корней алгебры $\mathfrak{g}$ с фиксированной подсистемой положительных корней $\mathrm{R}^{+} \subset \mathrm{R}$ и базисом простых корней $\Pi^{+} \subset \mathrm{R}^{+}$. Для каждого веса $\lambda \in \mathfrak{h}^{*}$ определим его образ $h_{\lambda}$ при действии изоморфизма $\mathfrak{h}^{*} \simeq \mathfrak{h}$, т. е. $(\lambda, \beta)=\beta\left(h_{\lambda}\right)$ для всех $\beta \in \mathfrak{h}^{*}$. Обозначим $\rho$ вектор Вейля $\left(\sum_{\alpha \in \mathrm{R}^{+}} \alpha\right) / 2$. 
Предположим, что $q \in \mathbb{C}$ не является корнем из единицы. Обозначим $U_{q}\left(\mathfrak{g}_{ \pm}\right)$ $\mathbb{C}$-алгебру, порожденную элементами $\left\{e_{ \pm \alpha}\right\}_{\alpha \in \Pi^{+}}$, которые удовлетворяют $q$-соотношениям Серра

$$
\sum_{k=0}^{1-a_{\alpha \beta}}(-1)^{k}\left[\begin{array}{c}
1-a_{\alpha \beta} \\
k
\end{array}\right]_{q_{\alpha}} e_{ \pm \alpha}^{1-a_{\alpha \beta}-k} e_{ \pm \beta} e_{ \pm \alpha}^{k}=0
$$

где $a_{\alpha \beta}=2(\alpha, \beta) /(\alpha, \alpha)$ - матрица Картана, $q_{\alpha}=q^{(\alpha, \alpha) / 2}$ и

$$
\left[\begin{array}{c}
m \\
k
\end{array}\right]_{q}=\frac{[m]_{q} !}{[k]_{q} ![m-k]_{q} !}, \quad[m]_{q} !=[1]_{q}[2]_{q} \ldots[m]_{q} .
$$

Здесь и далее $[z]_{q}=\left(q^{z}-q^{-z}\right) /\left(q-q^{-1}\right)$ всегда, когда величина $q^{ \pm z}$ имеет смысл.

Обозначим $U_{q}(\mathfrak{h})$ коммутативную $\mathbb{C}$-алгебру с генераторами $q^{ \pm h_{\alpha}}, \alpha \in \Pi^{+}$. Квантовая группа $U_{q}(\mathfrak{g})$ является $\mathbb{C}$-алгеброй, порожденной подалгебрами $U_{q}\left(\mathfrak{g}_{ \pm}\right)$и $U_{q}(\mathfrak{h})$ с соотношениями [16]

$$
q^{h_{\alpha}} e_{ \pm \beta} q^{-h_{\alpha}}=q^{ \pm(\alpha, \beta)} e_{ \pm \beta}, \quad\left[e_{\alpha}, e_{-\beta}\right]=\delta_{\alpha \beta} \frac{q^{h_{\alpha}}-q^{-h_{\alpha}}}{q_{\alpha}-q_{\alpha}^{-1}}
$$

Хотя $\mathfrak{h}$ не содержится в $U_{q}(\mathfrak{g})$, все же удобно сохранить ссылку на $\mathfrak{h}$.

Зафиксируем коумножение в $U_{q}(\mathfrak{g})$, как это сделано в работе [17]:

$$
\begin{gathered}
\Delta\left(e_{\alpha}\right)=e_{\alpha} \otimes q^{h_{\alpha}}+1 \otimes e_{\alpha}, \quad \Delta\left(e_{-\alpha}\right)=e_{-\alpha} \otimes 1+q^{-h_{\alpha}} \otimes e_{-\alpha}, \\
\Delta\left(q^{ \pm h_{\alpha}}\right)=q^{ \pm h_{\alpha}} \otimes q^{ \pm h_{\alpha}}
\end{gathered}
$$

для всех $\alpha \in \Pi^{+}$.

Подалгебры $U_{q}\left(\mathfrak{b}_{ \pm}\right) \subset U_{q}(\mathfrak{g})$, порожденные алгеброй $U_{q}\left(\mathfrak{g}_{ \pm}\right)$над $U_{q}(\mathfrak{h})$, являются квантованными универсальными обертывающими алгебрами подалгебр Бореля $\mathfrak{b}_{ \pm}=\mathfrak{h}+\mathfrak{g}_{ \pm} \subset \mathfrak{g}$. Отображение умножения осуществляет изоморфизм $U_{q}\left(\mathfrak{g}_{-}\right) \otimes$ $U_{q}\left(\mathfrak{g}_{+}\right) \otimes U_{q}(\mathfrak{h}) \rightarrow U_{q}(\mathfrak{g})$ векторных пространств, который также переходит в разложение $U_{q}\left(\mathfrak{b}_{ \pm}\right)=U_{q}\left(\mathfrak{g}_{ \pm}\right) U_{q}(\mathfrak{h})$.

Символ $n$ мы резервируем для обозначения ранга алгебры $\mathfrak{g}$. Пронумеруем элементы базиса $\Pi^{+}$так, чтобы $\mathfrak{g l}(n)$ являлась подалгеброй Ли в $\mathfrak{g}$ с простыми корнями $\left\{\alpha_{i}\right\}_{i=1}^{n-1}$, а $U_{q}(\mathfrak{g l}(n))$ - соответствующей квантовой подгруппой в $U_{q}(\mathfrak{g})$. Пусть $\mathfrak{g l}(\mathrm{s}) \subset \mathfrak{g l}(n)$ - максимальная подалгебра, устойчивая по отношению к автоморфизмам диаграммы Дынкина алгебры $\mathfrak{g}$. Имеем $\mathrm{s}=n$ для $\mathfrak{g}=\mathfrak{s p}(2 n), \mathfrak{g}=\mathfrak{s o}(2 n+1)$ и $\mathrm{s}=n-1$ для $\mathfrak{g}=\mathfrak{s o}(2 n)$.

Мы используем обозначения $e_{i}=e_{\alpha_{i}}$ и $f_{i}=e_{-\alpha_{i}}$ для $\alpha_{i} \in \Pi^{+}$во всех случаях, кроме $i=n, \mathfrak{g}=\mathfrak{s o}(2 n+1)$, для которого положим $f_{n}=[1 / 2]_{q} e_{-\alpha_{n}}$. Это преобразует соотношение (2.2) к виду

$$
\left[e_{n}, f_{n}\right]=\frac{q^{h_{\alpha_{n}}}-q^{-h_{\alpha_{n}}}}{q-q^{-1}} .
$$

Все остальные соотношения остаются неизменными.

2.1. Естественное представление. В этом разделе мы напоминаем естественное представление алгебры $\mathfrak{g}$ в векторном пространстве $\mathbb{C}^{N}$. Пусть $\left\{w_{i}\right\}_{i=1}^{N}-$ стандартный базис в пространстве $\mathbb{C}^{N}$. Используем обозначение $i^{\prime}=N+1-i$ для 
всех целых чисел $i \in I=[1, N]$, соответствующих отражению целочисленного интервала $I$ относительно центра $(N+1) / 2$. Для улучшения читаемости формул мы используем специальное обозначение $*=(N+1) / 2$.

Естественное представление строится следующим образом. Зададим матрицы

$$
\begin{gathered}
\pi\left(e_{i}\right)=E_{i, i+1}+E_{i^{\prime}-1, i^{\prime}}, \quad \pi\left(f_{i}\right)=E_{i+1, i}+E_{i^{\prime}, i^{\prime}-1}, \\
\pi\left(h_{\alpha_{i}}\right)=E_{i i}-E_{i+1, i+1}+E_{i^{\prime}-1, i^{\prime}-1}-E_{i^{\prime} i^{\prime}}
\end{gathered}
$$

при $i=1, \ldots, n-1$. Эти формулы определяют прямую сумму двух представлений подалгебры $U_{q}(\mathfrak{g l}(n))$. Расширим его до представления группы $U_{q}(\mathfrak{g})$ :

$$
\begin{gathered}
\pi\left(e_{n}\right)=E_{n, *}+E_{n^{\prime}-1, n^{\prime}}, \quad \pi\left(f_{n}\right)=E_{*, n}+E_{n^{\prime}, *}, \quad \pi\left(h_{\alpha_{n}}\right)=E_{n n}-E_{n^{\prime} n^{\prime}}, \\
\pi\left(e_{n}\right)=E_{n n^{\prime}}, \quad \pi\left(f_{n}\right)=E_{n^{\prime} n}, \quad \pi\left(h_{\alpha_{n}}\right)=2 E_{n n}-2 E_{n^{\prime} n^{\prime}}, \\
\pi\left(e_{n}\right)=E_{n-1, n^{\prime}}+E_{n, n^{\prime}+1}, \quad \pi\left(f_{n}\right)=E_{n^{\prime}, n-1}+E_{n^{\prime}+1, n}, \\
\pi\left(h_{\alpha_{n}}\right)=E_{n-1, n-1}+E_{n n}-E_{n^{\prime} n^{\prime}}-E_{n^{\prime}+1, n^{\prime}+1}
\end{gathered}
$$

соответственно для $\mathfrak{g}=\mathfrak{s o}(2 n+1), \mathfrak{g}=\mathfrak{s p}(2 n)$ и $\mathfrak{g}=\mathfrak{s o}(2 n)$. Подалгебра Картана представлена диагональными матрицами, а базисные элементы $w_{i}$ несут веса $\varepsilon_{i} \in \mathfrak{h}^{*}$, причем $\varepsilon_{i^{\prime}}=-\varepsilon_{i}$. Набор весов $\left\{\varepsilon_{i}\right\}_{i=1}^{n}$ образует ортонормированный базис $\mathfrak{h}^{*}$.

Введем частичное упорядочение в целочисленном интервале $[1, N]$, полагая $i \preccurlyeq j$ тогда и только тогда, когда $w_{j} \in U_{q}\left(\mathfrak{g}_{-}\right) w_{i}$. Имеем $i \prec j \Rightarrow i<j$.

Определим матрицу $F \in \operatorname{End}(V) \otimes U_{q}\left(\mathfrak{g}_{-}\right)$по формуле

$$
F=(\pi \otimes \mathrm{id})\left(q^{-\sum_{i=1}^{n} h_{\varepsilon_{i}} \otimes h_{\varepsilon_{i}}} \mathcal{R}\right) .
$$

Ее элементы $f_{i j}$, как показано ниже, выражаются через модифицированные коммутаторы $[x, y]_{a}=x y-a y x, a \in \mathbb{C}$. Для всех $\mathfrak{g}$ и $i<j \leqslant *$ положим

$$
f_{i j}=\left[f_{j-1}, \ldots,\left[f_{i+1}, f_{i}\right]_{\bar{q}}, \ldots\right]_{\bar{q}}, \quad f_{j^{\prime} i^{\prime}}=\left[\ldots,\left[f_{i}, f_{i+1}\right]_{\bar{q}}, \ldots, f_{j-1}\right]_{\bar{q}},
$$

где подразумевается, что $f_{i, i+1}=f_{i}=f_{i^{\prime}-1, i}$. Здесь и далее черта сверху означает обратную величину, например $\bar{q}=q^{-1}$. Кроме того,

- для $\mathfrak{g}=\mathfrak{s o}(2 n+1)$ справедливы соотношения $f_{n n^{\prime}}=(q-1) f_{n}^{2}$ и

$$
f_{i j^{\prime}}=q^{\delta_{i j}}\left[f_{*, j^{\prime}}, f_{i, *}\right]_{\bar{q}^{\delta_{i j}}}, \quad i, j<n
$$

- для $\mathfrak{g}=\mathfrak{s p}(2 n)$ справедливы соотношения $f_{n n^{\prime}}=[2]_{q} f_{n}$ и

$$
\begin{gathered}
f_{i n^{\prime}}=\left[f_{n}, f_{i n}\right]_{\bar{q}^{2}}, \quad f_{n i^{\prime}}=\left[f_{n^{\prime} i^{\prime}}, f_{n}\right]_{\bar{q}^{2}}, \quad i<n, \\
f_{i j^{\prime}}=q^{\delta_{i j}}\left[f_{n j^{\prime}}, f_{i n}\right]_{\bar{q}^{1+\delta_{i j}}}, \quad i, j<n
\end{gathered}
$$

- для $\mathfrak{g}=\mathfrak{s o}(2 n)$ справедливы соотношения $f_{n n^{\prime}}=0$ и

$$
\begin{gathered}
f_{i n^{\prime}}=\left[f_{n}, f_{i, n-1}\right]_{\bar{q}}, \quad f_{n i^{\prime}}=\left[f_{n^{\prime}+1, i^{\prime}}, f_{n}\right]_{\bar{q}}, \quad i<n-2, \\
f_{j i^{\prime}}=q^{\delta_{i j}}\left[f_{n i^{\prime}}, f_{j, n}\right]_{\bar{q}^{1+\delta_{i j}}}, \quad i, j<n .
\end{gathered}
$$

Наконец, $f_{i i}=1$ для всех $i$ и $f_{i j}=0$ при $i>j$. 
Матрица $F$ участвует в построении редуцированной обратной формы Шаповалова $\widehat{F}=\sum_{i, j=1}^{N} E_{i j} \otimes \hat{f}_{i j}$, которая получена ниже [9]. Удобно использовать язык

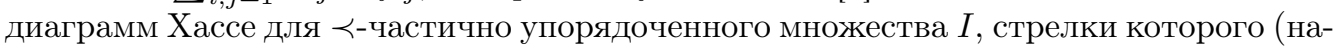
правленные к верхним узлам) помечены отрицательными генераторами Шевалле. Назовем любую возрастающую последовательность узлов $\left(m_{i}\right)_{i=1}^{k} \subset I$ путем от $m_{1}$ к $m_{k}$. Максимальный путь, т. е. тот, у которого соседние узлы соединены стрелками, называется траекторией. Если $i \prec m_{1}$ и $m_{k} \prec j$ для некоторых $i, j$, то будем писать $i \prec \vec{m} \prec j$.

Для всех $i, j \in I$ определим $\eta_{i j} \in \mathfrak{h}+\mathbb{C}$ по формуле

$$
\eta_{i j}=h_{\varepsilon_{i}}-h_{\varepsilon_{j}}+\rho_{i}-\rho_{j}-\frac{1}{2}\left\|\varepsilon_{i}-\varepsilon_{j}\right\|^{2},
$$

где $\rho_{i}=\left(\rho, \varepsilon_{i}\right)$, а $\|\beta\|^{2}$ - евклидова норма элемента $\beta \in \mathfrak{h}^{*}$. Будем считать $\eta_{i j}$ аффинной функцией $\mathfrak{h}^{*} \rightarrow \mathbb{C}, \lambda \mapsto\left(\lambda+\rho, \varepsilon_{i}-\varepsilon_{j}\right)-\left\|\varepsilon_{i}-\varepsilon_{j}\right\|^{2} / 2$. Элементы $\hat{f}_{i j}$ строятся следующим образом. При $i>j$ положим $\hat{f}_{i i}=1$ и $\hat{f}_{i j}=0$. При $i<j$ определим $A_{i}^{j}=\left(q-q^{-1}\right) /\left(q^{-2 \eta_{i j}}-1\right)$. Для пути $\vec{m}$ введем обозначения $f_{\vec{m}}=f_{m_{1}, m_{2}} \ldots f_{m_{k-1}, m_{k}}$ и $A_{\vec{m}}^{j}=A_{m_{1}}^{j} \ldots A_{m_{k}}^{j}$. Тогда

$$
\hat{f}_{i j}=\sum_{i \prec \vec{m} \prec j}^{\varnothing} f_{i, \vec{m}, j} A_{i, \vec{m}}^{j},
$$

где символ $\varnothing$ здесь и далее означает, что учитывается пустой путь $\vec{m}=\varnothing$. Элементы $\hat{f}_{1 j}$, где $j$ изменяется в интервале от двух до $N$ для алгебры $\mathfrak{g}=\mathfrak{s p}(N)$ и до $N-1$ для алгебры $\mathfrak{g}=\mathfrak{s o}(N)$, образуют набор отрицательных генераторов группы $Z_{q}\left(\mathfrak{g}, \mathfrak{g}^{\prime}\right)$, где $\mathfrak{g}^{\prime} \subset \mathfrak{g}-$ простая подалгебра Ли с корневым базисом $\left\{\alpha_{2}, \ldots, \alpha_{n}\right\}$.

Пусть $M_{\lambda}$ - модуль Верма со старшим весом $\lambda \in \mathfrak{h}^{*}$ и с каноническим генератором $v_{\lambda}$. Матрица $\widehat{F}$ рассматривается как отображение $\mathfrak{h}^{*} \mapsto E n d(V) \otimes U_{q}\left(\mathfrak{g}_{-}\right)$такое, что $\hat{f}_{i j}(\lambda) v_{\lambda}=\hat{f}_{i j} v_{\lambda}$. Тензоры $\widehat{F}_{j}=\widehat{F}\left(w_{j} \otimes v_{\lambda}\right)=\sum_{i=1}^{j} w_{i} \otimes \hat{f}_{i j} v_{\lambda}$ являются сингулярными векторами, т. е. аннигилируются всеми $e_{\alpha}, \alpha \in \Pi^{+}$. Они хорошо определены для $\lambda$ в общем положении и порождают подмодули $M_{j} \simeq M_{\lambda+\varepsilon_{j}} \subset V \otimes M_{\lambda}$. При некоторых весах $\widehat{F}_{j}$ имеют нули и полюсы и поэтому нуждаются в регуляризации, поскольку сингулярные векторы определены с точностью до скалярного множителя. В частности, существует естественная регуляризация $\check{f}_{i j}=\hat{f}_{i j} \prod_{l \prec j} \bar{A}_{l} \in U_{q}\left(\mathfrak{b}_{-}\right)$. Оказывается, что она чрезмерна в определенных случаях, таких как наличие нулей при некоторых $\lambda$. Мы изучаем этот вопрос для наиболее важных пар $(i, j)$, связанных с генераторами группы $Z_{q}\left(\mathfrak{g}, \mathfrak{g}^{\prime}\right)$.

\section{3. СТАНДАРТНАЯ ФИЛЬТРАЦИЯ В $V \otimes M_{\lambda}$}

Изучение матрицы $\widehat{F}$ основано на анализе тензорного произведения $V \otimes M_{\lambda}$. Его модульная структура в большой степени описывается $U_{q}(\mathfrak{g})$-инвариантным оператором $\mathcal{Q}=(\pi \otimes \mathrm{id})\left(\mathcal{R}_{21} \mathcal{R}\right) \in \operatorname{End}(V) \otimes M_{\lambda}$, который является скаляром на подмодулях и фактормодулях старшего веса. Обозначая через $x_{j}$ его собственное значение на подмодуле $M_{j}$, имеем $x_{i} x_{j}^{-1}=\left.q^{2 \xi_{i j}}\right|_{\lambda}$, где

$$
\xi_{i j}=h_{\varepsilon_{i}}-h_{\varepsilon_{j}}+\rho_{i}-\rho_{j}+\frac{1}{2}\left(\left\|\varepsilon_{i}\right\|^{2}-\left\|\varepsilon_{j}\right\|^{2}\right) .
$$

Для $\lambda$ в общем положении числа $x_{j}$ попарно различны и разделяют подмодули $M_{j}$. 
Другим средством анализа тензорного произведения $V \otimes M_{\lambda}$ является последовательность подмодулей $\left(V_{j}\right)_{j=1}^{N}$, порожденная генераторами $v_{\lambda, l}=w_{l} \otimes v_{\lambda}, l=1, \ldots, j$. Она формирует возрастающую фильтрацию произведения $V \otimes M_{\lambda}$, чей градуированный модуль изоморфен прямой сумме $\oplus_{j=1}^{N} V_{j} / V_{j-1}$ модулей Верма. Отсюда следует, что все $V_{j}$ являются $\mathcal{Q}$-инвариантными (см. статью [18]). В настоящем разделе изучается проекция $\wp_{j}: M_{j} \rightarrow V_{j} / V_{j-1}$, которая может либо быть нулевой либо являться изоморфизмом. Из весовых соображений следует, что $M_{j} \subset V_{j}$, а сингулярный вектор $\widehat{F}_{j}$ отображается на линию, порожденную $v_{\lambda, j} \bmod V_{j-1}$, старшим вектором фактора $V_{j} / V_{j-1}$. Введем $\widehat{C}_{i}$ по формуле $\wp_{j}\left(\widehat{F}_{j}\right)=\widehat{C}_{j} v_{\lambda, j}$. Нашей ближайшей целью является вычисление $\widehat{C}_{j}, j=1, \ldots, N$. Ясно, что $\widehat{C}_{1}=1$. Для $j>1$ ответ дается в рассмотренном далее предложении 3.2 .

Для всех $j \in I$ введем коммутативную алгебру $\mathcal{A}_{j}$ следующим образом. Если $j \leqslant *$, то положим $\mathcal{A}_{j}=\mathbb{C}\left[y_{1}^{ \pm 1}, \ldots, y_{j-1}^{ \pm 1}\right]$. В противном случае $\mathcal{A}_{j}$ является фактором алгебры $\mathbb{C}\left[y_{1}^{ \pm 1}, \ldots, y_{j-1}^{ \pm 1}\right]$ по модулю соотношений $y_{l} y_{l^{\prime}}=y_{j^{\prime}}, l=j^{\prime}+1, \ldots, j-1$, а для $\mathfrak{g}=\mathfrak{s o}(2 n+1)$ дополним ее элементом $y_{j^{\prime}}^{1 / 2}$ с соотношением $q y_{*}=y_{j^{\prime}}^{1 / 2}$. Во всех случаях $\mathcal{A}_{j}$ является локализацией полиномиальной алгебры. Алгебру $\mathcal{A}_{j}$ можно реализовать как подалгебру в $\widehat{U}_{q}(\mathfrak{h})$, положив в ней $y_{l}=q^{-2 \eta_{l j}}, l \prec j$, благодаря следующему факту.

ПреДЛОЖЕНИЕ 3.1. Для всех $m=2, \ldots, n$ имеем $\eta_{m 1^{\prime}}+\eta_{m^{\prime} 1^{\prime}}=\eta_{11^{\prime}}$. Также для $\mathfrak{g}=\mathfrak{s o}(2 n+1)$ имеем $2 \eta_{*, m^{\prime}}-1=\eta_{m, m^{\prime}}, m=1, \ldots, n$.

ДОКАЗАТЕЛЬСТво очевидно.

Будем считать $\hat{f}_{i j}$ элементом свободного $\mathcal{A}_{j}$-модуля, порожденного подалгеброй $U_{q}\left(\mathfrak{g}_{-}\right)$. Рассмотрим $\widehat{C}_{j}$ как полином по $B_{i}=-A_{i}, i=1, \ldots, j-1$, в котором $A_{i}=(q-\bar{q}) /\left(y_{i}-1\right)$. Пусть $\|i-j\|$ означает число стрелок на диаграмме Хассе, расположенных на траектории от $i$ до $j$.

ПрЕДЛОЖЕНИЕ 3.2. Коэффициенты $\widehat{C}_{j}, j=2, \ldots, N$, допускают следующую факторизацию:

$$
\widehat{C}_{j}= \begin{cases}\left(1-[2]_{q} q^{2} A_{j}\right) \prod_{\substack{i=1, i \neq j^{\prime}}}^{j-1}\left(1-q A_{i}\right), & \mathfrak{g}=\mathfrak{s p}(N), \\ \frac{y_{*}-q}{y_{*}-\bar{q}} \prod_{\substack{i=1, i \neq j^{\prime}, *}}^{j-1}\left(1-q A_{i}\right), & \mathfrak{g}=\mathfrak{s o}(2 n+1), \\ \prod_{i=1,}^{j-1}\left(1-q A_{i}\right), & \mathfrak{g}=\mathfrak{s o}(2 n), \\ i \neq j^{\prime} & \end{cases}
$$

где множитель $\left(y_{*}-q\right)\left(y_{*}-\bar{q}\right)$ присутствует только при $*<j$.

Доказательство основано на понятии главного монома, который связан с каждой парой $i \prec j$. Каждая траектория от $i$ к $j$ порождает единственный элемент $\psi_{j i} \in U_{q}\left(\mathfrak{g}_{-}\right)$такой, что $w_{j}=\psi_{j i} w_{i}$. Обозначим $\psi^{i j}$ элемент, полученный из $\psi_{j i}$ обращением порядка простых множителей, и назовем его главным мономом пары $(i, j)$. 
ЛЕМма 3.1 (СМ. СТАТЬЮ [18]). Предположим, что $i \prec j u \psi \in U_{q}\left(\mathfrak{g}_{-}\right)$является мономом Шевалле веса $\varepsilon_{j}-\varepsilon_{i}$, отличным от $\psi^{i j}$. Тогда $\wp_{j}(\psi)=0$. Кроме того, $\wp_{j}\left(w_{i} \otimes \psi^{i j} v_{\lambda}\right)=(-1)^{|i-j|} q^{\tilde{\rho}_{i}-\tilde{\rho}_{j}} v_{\lambda, j}$, где $\tilde{\rho}_{i}=\rho_{i}+\left\|\varepsilon_{i}\right\|^{2} / 2$.

ДокАзАтЕльство. Первая часть утверждения доказана в работе [18] (см. лемму 3.4). Аналогичное утверждение для другой версии коумножения доказано там же. Здесь мы даем доказательство для используемой версии квантовой группы. Предположим, что $\alpha \in \Pi^{+}$и $\varepsilon_{i}-\varepsilon_{k}=\alpha$. Согласно лемме 3.4 статьи [18] узел $w_{i} \otimes \psi^{k j} v_{\lambda}$ лежит в $V_{j-1}$. Применяя $\Delta f_{\alpha}=f_{\alpha} \otimes 1+q^{-h_{\alpha}} \otimes f_{\alpha}$ к $w_{i} \otimes \psi^{k j} v_{\lambda}$, получаем, что для всех $k \preccurlyeq j$

$$
w_{i} \otimes \psi^{i j} v_{\lambda}=-q^{\left(\alpha, \varepsilon_{i}\right)} w_{k} \otimes \psi^{k j} v_{\lambda}=-q^{\tilde{\rho}_{i}-\tilde{\rho}_{k}} w_{k} \otimes \psi^{k j} v_{\lambda} \quad \bmod V_{j-1}
$$

Здесь использованы соотношения $f_{\alpha} w_{i}=w_{k}$ и $f_{\alpha} \psi^{k j}=\psi^{i j}$ для всех $k \preccurlyeq j$. Продолжая процедуру вдоль траектории от $i$ к $j$, завершаем доказательство.

Благодаря лемме 3.1 только главные мономы $\hat{f}_{i j}$ вносят вклад в $\wp_{j}\left(w_{j} \otimes v_{\lambda}\right)$. Положим $\sigma=1$ для симплектической алгебры $\mathfrak{g}$ и $\sigma=-1$ для ортогональной $\mathfrak{g}$.

ЛЕмма 3.2. Для элемента $f_{i j}(i, j)$-главный моном равен $(-1)^{|i-j|-1} c_{i j} \psi^{i j}$, где

$$
c_{i j}= \begin{cases}\bar{q}^{\eta_{i j}(0)}, & i \neq j^{\prime}, \\ \bar{q} \bar{q}^{\eta_{i j}(0)}+\sigma q, & i=j^{\prime} .\end{cases}
$$

\section{ДокАЗАТЕЛЬСТво очевидно.}

Для данного пути $\vec{m}=\left(m_{1}, \ldots, m_{k}\right)$ положим $c_{\vec{m}}=c_{m_{1}, m_{2}} \ldots c_{m_{k-1}, m_{k}}$. Введем числа $\hat{c}_{i j} \in \mathbb{C}$ с помощью равенства $\wp_{j}\left(w_{i} \otimes \hat{f}_{i j} v_{\lambda}\right)=\hat{c}_{i j} v_{\lambda, j}$, так что $\widehat{C}_{j}=\sum_{i=1}^{j} \hat{c}_{i j}$. Легко проверить, что $\hat{c}_{i j}=\sum_{i \prec \vec{m} \prec j}^{\varnothing} c_{i, \vec{m}, j} B_{i, \vec{m}} q^{\tilde{\rho}_{i}-\tilde{\rho}_{j}}$.

ДоКАЗАТЕЛЬСТво ПРЕДЛОЖЕНИЯ 3.2 ДЛЯ $j=N$. Докажем этот частный случай индукцией по $N$. Базис для индукции получаем сразу: $\widehat{C}_{2}=1$ для $\mathfrak{g}=\mathfrak{s o}(2)$. Менее очевидно, хотя и непосредственно доказывается, что для $\mathfrak{g}=\mathfrak{s o}(3) \widehat{C}_{3}=$ $\left(y_{*}-q\right) /\left(y_{*}-\bar{q}\right)$ и для $\mathfrak{g}=\mathfrak{s p}(2) \widehat{C}_{2}=1-[2]_{q} q^{2} A_{1}$. Итак, положим далее $\varepsilon_{2} \neq 0$ для высших $N$. Наша стратегия заключается в выделении $\phi_{i}=B_{i}+q^{-1}$ при $i=2^{\prime}, 2$. Это облегчит индукционный переход.

Учитывая условие $1+\hat{c}_{2^{\prime} 1^{\prime}}=1+B_{2^{\prime}} q^{\tilde{\rho}_{2^{\prime}}-\tilde{\rho}_{1^{\prime}}}=q \phi_{2^{\prime}}$, вычислим далее $\sum_{i=3}^{2^{\prime}} \hat{c}_{i 1^{\prime}}$. Заметим, что для всех $3 \prec \vec{m} \prec 2^{\prime}$ справедливо равенство $c_{i, \vec{m}, 1^{\prime}}=q^{-1} c_{i, \vec{m}, 2^{\prime}, 1^{\prime}}=$ $q^{-1} c_{i, \vec{m}, 2^{\prime}}$. Кроме того, сделаем следующую замену для всех $i: q^{\tilde{\rho}_{i}-\tilde{\rho}_{1^{\prime}}}=q q^{\tilde{\rho}_{i}-\tilde{\rho}_{2^{\prime}}}$. Тогда

$$
\begin{aligned}
1+\sum_{i=3}^{2^{\prime}} \hat{c}_{i 1^{\prime}} & =q \phi_{2^{\prime}}+\sum_{i=3}^{3^{\prime}} \sum_{i \prec \vec{m} \prec 2^{\prime}}^{\varnothing} c_{i, \vec{m}, 2^{\prime}, 1^{\prime}} B_{i, \vec{m}} B_{2^{\prime}} q^{\tilde{\rho}_{i}-\tilde{\rho}_{1^{\prime}}}+\sum_{i=3}^{3^{\prime}} \sum_{i \prec \vec{m} \prec 2^{\prime}}^{\varnothing} c_{i, \vec{m}, 1^{\prime}} B_{i, \vec{m}} q^{\tilde{\rho}_{i}-\tilde{\rho}_{1^{\prime}}}= \\
& =q \phi_{2^{\prime}}\left(1+\sum_{i=3}^{3^{\prime}} \sum_{i \prec \vec{m} \prec 2^{\prime}}^{\varnothing} c_{i, \vec{m}, 2^{\prime}} B_{i, \vec{m}} q^{\tilde{\rho}_{i}-\tilde{\rho}_{2^{\prime}}}\right) .
\end{aligned}
$$


Теперь сумма $\sum_{i=1}^{2} \hat{c}_{i 1^{\prime}}$ приобретает вид

$$
\begin{aligned}
\sum_{i=1}^{2} \hat{c}_{i 1^{\prime}} & =\sum_{i=1}^{2} \sum_{i \prec \vec{m} \prec 1^{\prime}}^{\varnothing} c_{i, \vec{m}, 1^{\prime}} B_{i, \vec{m}} q^{\tilde{\rho}_{i}-\tilde{\rho}_{1^{\prime}}}= \\
& =\sum_{i=1}^{2} \sum_{i \prec \vec{m} \prec 2^{\prime}}^{\varnothing}\left(c_{i, \vec{m}, 2^{\prime}, 1^{\prime}} B_{i, \vec{m}, 2^{\prime}} q^{\tilde{\rho}_{i}-\tilde{\rho}_{1^{\prime}}}+c_{i, \vec{m}, 1^{\prime}} B_{i, \vec{m}} q^{\tilde{\rho}_{i}-\tilde{\rho}_{1^{\prime}}}\right)= \\
& =\phi_{2^{\prime}} \sum_{i=1}^{2} \sum_{i \prec \vec{m} \prec 2^{\prime}}^{\varnothing} c_{i, \vec{m}, 2^{\prime}} B_{i, \vec{m}} q^{\tilde{\rho}_{i}-\tilde{\rho}_{1^{\prime}}}+\sum_{i=1}^{2} \sum_{i \prec \vec{m} \prec 2^{\prime}}^{\varnothing}\left(c_{i, \vec{m}, 1^{\prime}}-q^{-1} c_{i, \vec{m}, 2^{\prime}}\right) B_{i, \vec{m}} q^{\tilde{\rho}_{i}-\tilde{\rho}_{1^{\prime}}} .
\end{aligned}
$$

В последней сумме ненулевыми являются только те разности, которые соответствуют $i=1, \vec{m}=\varnothing, \vec{m}=(2)$ и $i=2, \vec{m}=\varnothing$. Они соответственно равны

$$
c_{1,1^{\prime}}-q^{-1} c_{1,2^{\prime}}=q^{-\theta_{1,1^{\prime}}-1}+\sigma q-q^{-1} q^{-\theta_{1,2^{\prime}}}=\sigma q
$$

И

$$
c_{1,2,1^{\prime}}-q^{-1} c_{1,2,2^{\prime}}=c_{2,1^{\prime}}-q^{-1} c_{2,2^{\prime}}=q^{-\theta_{2,1^{\prime}}}-\sigma-q^{-1} q^{-\theta_{2,2^{\prime}}-1}=-\sigma .
$$

Отсюда получаем последний член

$$
\sigma q\left(q B_{1}-B_{1,2}-B_{2} q^{\tilde{\rho}_{2}-\tilde{\rho}_{1}}\right) q^{\tilde{\rho}_{1}-\tilde{\rho}_{2^{\prime}}}=\sigma q \phi_{2^{\prime}}\left(B_{1}-B_{2}\right) q^{\tilde{\rho}_{1}-\tilde{\rho}_{2^{\prime}}},
$$

поскольку $\tilde{\rho}_{2}-\tilde{\rho}_{1}=\rho_{2}-\rho_{1}=-1$. Таким образом, мы вынесли за скобки множитель $q \phi_{2^{\prime}}$ в выражении для $\widehat{C}_{1^{\prime}}$ :

$$
\frac{\widehat{C}_{1^{\prime}}}{q \phi_{2^{\prime}}}=1+\sum_{i=1}^{3^{\prime}} \sum_{i \prec \vec{m} \prec 2^{\prime}}^{\varnothing} c_{i, \vec{m}, 2^{\prime}} B_{i, \vec{m}} q^{\tilde{\rho}_{i}-\tilde{\rho}_{2^{\prime}}}+\sigma\left(B_{1}-B_{2}\right) q^{\tilde{\rho}_{1}-\tilde{\rho}_{2^{\prime}}} .
$$

Отдельно рассмотрим суммы по $i=1,2$ и $3 \leqslant i \leqslant 3^{\prime}$. Сначала, используя равенство

$$
c_{12^{\prime}}-q^{-1} c_{22^{\prime}}=q^{-\theta_{1,2^{\prime}}}-\sigma-q^{-\theta_{2,2^{\prime}}-2}=-\sigma,
$$

вычислим внутреннюю сумму при $i=1$ :

$$
\begin{aligned}
& \sum_{2 \prec \vec{m} \prec 2^{\prime}}^{\varnothing}\left(c_{1,2, \vec{m}, 2^{\prime}} B_{1, \vec{m}} B_{2}+c_{1, \vec{m}, 2^{\prime}} B_{1, \vec{m}}\right) p^{\tilde{\rho}_{1}-\tilde{\rho}_{2^{\prime}}}= \\
& =q \phi_{2} \sum_{2 \prec \vec{m} \prec 2^{\prime}}^{\varnothing} c_{2, \vec{m}, 2^{\prime}} B_{1, \vec{m}} q^{\tilde{\rho}_{2}-\tilde{\rho}_{2^{\prime}}}-\sigma B_{1} q^{\tilde{\rho}_{1}-\tilde{\rho}_{2^{\prime}}},
\end{aligned}
$$

где использована подстановка $q^{\tilde{\rho}_{1}-\tilde{\rho}_{2^{\prime}}}=q q^{\tilde{\rho}_{2}-\tilde{\rho}_{2^{\prime}}}$.

Теперь заметим, что для $3 \leqslant k \leqslant 3^{\prime}$ и $l=1,2$ справедливы соотношения

$$
c_{l k}=q^{-\theta_{l k}}=q^{-\rho_{l}+\rho_{k}+\left\|\varepsilon_{l}-\varepsilon_{k}\right\|^{2} / 2}=q^{-\tilde{\rho}_{l}+\tilde{\rho}_{k}+\left\|\varepsilon_{l}-\varepsilon_{k}\right\|^{2} / 2+\left(\left\|\varepsilon_{l}\right\|^{2}-\left\|\varepsilon_{k}\right\|^{2}\right) / 2}=q^{-\tilde{\rho}_{l}+\tilde{\rho}_{k}+1},
$$

поскольку по нашему предположению $\varepsilon_{2} \neq 0$. Перепишем внутреннюю сумму для $i=2$ в формуле (3.2) следующим образом:

$$
c_{22^{\prime}} B_{2} q^{\tilde{\rho}_{2}-\tilde{\rho}_{2^{\prime}}}+\sum_{k=3}^{3^{\prime}} \sum_{k \prec \vec{m} \prec 2^{\prime}}^{\varnothing} c_{k, \vec{m}, 2^{\prime}} B_{k, \vec{m}} B_{2} q^{\tilde{\rho}_{k}-\tilde{\rho}_{2^{\prime}}+1} .
$$


Вместе с суммированием по $3 \leqslant i \leqslant 3^{\prime}$ в $(3.2)$ это приводит к выражению

$$
-c_{22^{\prime}} q^{\tilde{\rho}_{2}-\tilde{\rho}_{2^{\prime}}}+\phi_{2} c_{22^{\prime}} q^{\tilde{\rho}_{2}-\tilde{\rho}_{2^{\prime}}}+\phi_{2} \sum_{i=3}^{3^{\prime}} \sum_{i \prec \vec{m} \prec 2^{\prime}}^{\varnothing} c_{i, \vec{m}, 2^{\prime}} B_{i, \vec{m}} q^{\tilde{\rho}_{i}-\tilde{\rho}_{2^{\prime}}+1} .
$$

После таких преобразований формула (3.2) принимает вид

$$
\begin{aligned}
\frac{\widehat{C}_{1^{\prime}}}{q \phi_{2^{\prime}}}= & 1-c_{22^{\prime}} q^{\tilde{\rho}_{2}-\tilde{\rho}_{2^{\prime}}-1}-\sigma\left(\phi_{2}-q^{-1}\right) q^{\tilde{\rho}_{1}-\tilde{\rho}_{2^{\prime}}}+\phi_{2} c_{22^{\prime}} q^{\tilde{\rho}_{2}-\tilde{\rho}_{2^{\prime}}}+ \\
& +q \phi_{2} \sum_{i=3}^{3^{\prime}} \sum_{i \prec \vec{m} \prec 2^{\prime}}^{\varnothing} c_{i, \vec{m}, 2^{\prime}} B_{i, \vec{m}} q^{\tilde{\rho}_{i}-\tilde{\rho}_{2^{\prime}}}+q \phi_{2} \sum_{2 \prec \vec{m} \prec 2^{\prime}}^{\varnothing} c_{2, \vec{m}, 2^{\prime}} B_{1, \vec{m}} q^{\tilde{\rho}_{2}-\tilde{\rho}_{2^{\prime}}} .
\end{aligned}
$$

Поскольку

$$
-\sigma q^{\tilde{\rho}_{1}-\tilde{\rho}_{2^{\prime}}}+c_{22^{\prime}} q^{\tilde{\rho}_{2}-\tilde{\rho}_{2^{\prime}}}=-\sigma q^{\tilde{\rho}_{1}-\tilde{\rho}_{2^{\prime}}}+\left(q^{-\theta_{22^{\prime}}-1}+\sigma q\right) q^{\tilde{\rho}_{2}-\tilde{\rho}_{2^{\prime}}}=q^{-\theta_{22^{\prime}}-1} q^{\tilde{\rho}_{2}-\tilde{\rho}_{2^{\prime}}}=q,
$$

первая строка равна $q \phi_{2}$. В конечном итоге мы приходим к равенству

$$
\frac{\widehat{C}_{1^{\prime}}}{\phi_{2} \phi_{2^{\prime}} q^{2}}=1+\sum_{i=2}^{3^{\prime}} \sum_{i \prec \vec{m} \prec 2^{\prime}}^{\varnothing} c_{i, \vec{m}, 2^{\prime}} B_{i, \vec{m}} q^{\tilde{\rho}_{i}-\tilde{\rho}_{2^{\prime}}} .
$$

Правая сторона равна в точности $\widehat{C}_{2^{\prime}}\left(y_{1}, y_{3}, \ldots, y_{3^{\prime}}\right)$ при $\operatorname{dim} V=N-2$. Отсюда получаем $\widehat{C}_{1^{\prime}}$ методом индукции по $N$. Предложение доказано для $j=N$.

ДОКАЗАТЕЛЬСТВО ПРЕДЛОЖЕНИЯ 3.2 ДЛЯ $j$ ОБЩЕГО ПОЛОЖЕНИЯ. Теперь предположим, что $1<j^{\prime} \leqslant j$. Пусть $k$ равно $j+1$, тогда $k^{\prime}=j^{\prime}-1$. Используя формулу $c_{1 i}=q^{\tilde{\rho}_{i}-\tilde{\rho}_{1}+1}$ при $1<i<j^{\prime}-1$, представим $\hat{c}_{1 j}=\sum_{1 \prec \vec{m} \prec j^{\prime}}^{\varnothing} c_{1, \vec{m}} B_{1, \vec{m}} q^{\tilde{\rho}_{1}-\tilde{\rho}_{j}}$ как

$$
c_{1 j} q^{\tilde{\rho}_{1}-\tilde{\rho}_{j}}+B_{1} \sum_{i=2}^{j-1} \sum_{i \prec \vec{m} \prec j}^{\varnothing} c_{1 i} c_{i, \vec{m}} B_{i, \vec{m}} q^{\tilde{\rho}_{1}-\tilde{\rho}_{j}}=q B_{1}\left(1+\sum_{i=2}^{j-1} \sum_{i \prec \vec{m} \prec j^{\prime}}^{\varnothing} c_{i, \vec{m}} B_{i, \vec{m}} q^{\tilde{\rho}_{i}-\tilde{\rho}_{j}}\right) .
$$

Это выражение равно $q B_{1}\left(1+\sum_{i=2}^{j-1} \hat{c}_{i j}\right)$, но также и

$$
\widehat{C}_{j}=1+\sum_{i=2}^{j-1} \hat{c}_{i j}+\hat{c}_{1 j}=q B_{1}\left(1+\sum_{i=2}^{j-1} \hat{c}_{i j}\right)
$$

По индукции получаем, что $\widehat{C}_{j}=q B_{1} \ldots q B_{j^{\prime}-1}\left(1+\sum_{i=j^{\prime}}^{j-1} \hat{c}_{i j}\right)$. Выражение в скобках есть не что иное, как $\widehat{C}_{j}\left(y_{j^{\prime}}, y_{j^{\prime}+1}, \ldots, y_{j-1}\right)$ при $\operatorname{dim} V=j$. Его факторизация уже доказана. Предложение доказано для $j$ общего положения.

Применим предложение 3.2 к анализу элементов матрицы $\check{f}_{i j}$ для ортогональной алгебры $\mathfrak{g}$. Введем $\delta_{j}^{-} \in \mathcal{A}_{j}$ следующим образом. Положим $\delta_{j}^{-}=1$ для $j<\mathrm{s}^{\prime}$, а для $j \geqslant \mathrm{~s}^{\prime}$ положим $\delta_{j}^{-}=\left(y_{j^{\prime}}-1\right) /(q-\bar{q})=\bar{A}_{j^{\prime}}$, если $\mathfrak{g}=\mathfrak{s o}(2 n)$, и $\delta_{j}^{-}=\left(y_{j^{\prime}}^{1 / 2}+1\right) /(q+1)$, если $\mathfrak{g}=\mathfrak{s o}(2 n+1)$. В случае симплектической $\mathfrak{g}$ положим $\delta_{j}^{-}=1$ для всех $j$.

ЛЕмма 3.3. Предположим, что $\mathfrak{g}=\mathfrak{s o}(N)$. Для всех $i, j$, удовлетворяющих условию $i \leqslant j^{\prime}$, элемент $\check{f}_{i j}$ делится на $\delta_{j}^{-}$. 
ДокАЗАтЕльство. Требуется рассмотреть только случай $i \leqslant \mathrm{~s}, \mathrm{~s}^{\prime} \leqslant j$. Без ограничения общности положим $i=1$. Вспомним базисное разложение

$$
\widehat{F}_{j}=\sum_{l=1}^{j} w_{l} \otimes \hat{f}_{l j} v_{\lambda}
$$

и рассмотрим сингулярный вектор

$$
\widehat{F}_{j}^{\sharp}=\frac{y_{j^{\prime}}-1}{q-\bar{q}} \widehat{F}_{j} .
$$

Для специального выбора $\left.q^{-2 \eta_{l 1^{\prime}}}\right|_{\lambda}=y_{l}, l \prec j$, имеем $\hat{f}_{1 j}(\lambda)=\hat{f}_{1 j}^{\sharp}(q-\bar{q}) /\left(y_{j^{\prime}}-1\right)$. Заметим, что $y_{j^{\prime}}-1$ делится на $\delta_{j}^{-}$, а $A_{j^{\prime}}=(q-\bar{q}) /\left(y_{j^{\prime}}-1\right)$ является единственным множителем в $\hat{f}_{1 j}$, имеющим полюс в точке $\delta_{j}^{-}=0$, поэтому $\widehat{F}_{j}^{\sharp}$ регулярна в точке $\delta_{j}^{-}=0$. Поскольку $\widehat{C}_{j}$ является регулярной в $\lambda$ общего положения при условии $\delta_{j}^{-}=0$, имеем равенство $\wp_{j}\left(\widehat{F}_{j}^{\sharp}\right)=0$. Это возможно только в том случае, если $\widehat{F}_{j}^{\sharp}=0$ или $\widehat{F}_{j}^{\sharp} \in V_{j-1}$. Из всех $\mathcal{Q}$-собственных чисел только $x_{j}, x_{j^{\prime}}$ для четных $N$ и $x_{j}, x_{*}, x_{j^{\prime}}$ для нечетных $N$ становятся постоянными при $\delta_{j}^{-}=0$, а остальные можно сделать отличными от $x_{j}$. Поскольку $x_{j} x_{j^{\prime}}^{-1}=q^{-4} y_{j^{\prime}} \neq 1$ и $x_{j} x_{*}^{-1}=q^{-1} y_{j^{\prime}}^{1 / 2} \neq 1$, спектр оператора $\mathcal{Q}$, ограниченного на $V_{j-1}$, не содержит $x_{j}$ - свое собственное значение на $M_{j}$. Поэтому $\widehat{F}_{j}^{\sharp}$ обращается в ноль вместе со своим коэффициентом $\hat{f}_{1 j}^{\sharp}$ при условии $\delta_{j}^{-}=0$. Следовательно, $\check{f}_{1 j}$ делится на $\delta_{j}^{-}$. Лемма доказана.

\section{4. РЕГУЛЯРИЗАЦИЯ МАТРИЧНЫХ КОЭФФИЦИЕНТОВ}

В данном разделе мы описываем регуляризацию элемента $\hat{f}_{i j}$. Рассмотрим редукцию $\check{f}_{i j}$ вдоль двусторонних идеалов в $\mathcal{B}=U_{q}\left(\mathfrak{g}_{-}\right)$из следующего семейства. Обозначим $\mathfrak{S}$ объединение целых интервалов $[1, \mathrm{~s}] \cup\left[\mathrm{s}^{\prime}, 1^{\prime}\right]$ и $\left.\mathfrak{S}_{i j}=\right] i, j[\cap \mathfrak{S}$ для всех пар $i \prec j$. Зафиксируем $k \in \mathfrak{S}$, пусть также $l, r-$ ближайшие узлы к узлу $k$, такие что $l \prec k \prec r$. Предположим, что $f_{\alpha}$ и $f_{\beta}$, соответствующие $\alpha=\varepsilon_{k}-\varepsilon_{l}$ и $\beta=\varepsilon_{r}-\varepsilon_{k}$, не коммутируют. Определим двусторонний идеал $J_{k} \subset \mathcal{B}$, порожденный всеми такими $f_{l r}$ (в точности один, если только не выполняются условия $\mathfrak{g}=\mathfrak{s o}(2 n)$ и $\left.k=\mathrm{s}, \mathrm{s}^{\prime}\right)$. Как следует из соотношений $(2.1), \mathcal{B} / J_{k}$ изоморфно $U_{q}\left(\mathfrak{g}_{-}^{1}\right) \otimes U_{q}\left(\mathfrak{g}_{-}^{2}\right)$ как векторное пространство, где $\mathfrak{g}^{i} \subset \mathfrak{g}$ - некоторые подалгебры Ли. Справедливо равенство $f_{\beta} f_{\alpha}=\bar{q} f_{\alpha} f_{\beta}$ во всех случаях, кроме $\mathfrak{g}=\mathfrak{s p}(2 n)$ и $k=n, n^{\prime}$; тогда $f_{\beta} f_{\alpha}=\bar{q}^{2} f_{\alpha} f_{\beta}$. Кроме того, верны следующие утверждения.

Лемма 4.1. Для всех $i \prec j u k \in \mathfrak{S}_{i j}$ справедливы равенства $f_{i j}=0$ по модулю $J_{k} u f_{i j}=(q-\bar{q}) f_{i k} f_{k j}$ по модулю $J_{k^{\prime}}$.

ДокАЗАТЕЛЬство легко следует из формул (2.3)-(2.8).

СлеДСтвИЕ 4.1. Предположим, что $i \prec j$. Тогда

1) для всех $k \in \mathfrak{S}_{i j} \check{f}_{i j}=\check{f}_{i k} \check{f}_{k j} \bmod J_{k} u \check{f}_{i j}=\check{f}_{i k} \check{f}_{k j} y_{k} \bmod J_{k^{\prime}}$;

2) если $k, k^{\prime} \in \mathfrak{S}_{i j} u k<k^{\prime}, \operatorname{moz} \partial a \check{f}_{i j}=\check{f}_{i k} \check{f}_{k k^{\prime}} \check{f}_{k^{\prime} j} y_{k^{\prime}} \bmod J_{k}$.

ДоказАтЕЛьство. Проверим сначала первое утверждение. Зафиксируем $k \in \mathfrak{S}_{i j}$ и запишем выражение

$$
\hat{f}_{i j}=\sum_{i \prec \vec{m} \prec k \prec \vec{l} \prec j}^{\varnothing}\left(f_{i, \vec{m}, k} A_{i, \vec{m}} f_{k, \vec{l}, j} A_{\vec{l}} A_{k}+f_{i, \vec{m}, \vec{l}, j} A_{i, \vec{m}} A_{\vec{l}}\right) .
$$


Вторая сумма обращается в ноль по модулю $J_{k}$, а это влечет существование первой факторизации. Элемент $f_{i, \vec{m}, \vec{l}, j}$ факторизуется как $\left(q-q^{-1}\right) f_{i, \vec{m}, k} f_{k, \vec{l}, j}$ по модулю $J_{k^{\prime}}$ согласно лемме 4.1. Учитывая, что $\left(A_{k}+q-\bar{q}\right) \bar{A}_{k}=y_{k}$, получаем факторизацию по модулю $J_{k^{\prime}}$. Наконец, заметим, что второе утверждение следует из первого. Следствие доказано.

СлеДСтвиЕ 4.2. Элементы $\check{f}_{i j}(\lambda)$ nри $i \prec j<\mathrm{s}^{\prime}$ или $\mathrm{s}<i \prec j$ не обращаются в нуль при всех $\lambda$.

ДокАзАтельство. Действительно, проецируя $\check{f}_{i j}$ вдоль $\sum_{k \in \mathfrak{S}_{i j}} J_{k}$, получаем $f_{\vec{m}}$, где $\vec{m}$ - (единственная) траектория из $i$ в $j$. Все множители в произведении являются генераторами Шевалле, поэтому $f_{\vec{m}}$ и, следовательно, $\check{f}_{i j}$ не обращаются в нуль. Следствие доказано.

Теорема 4.1. Предположим, что $i \prec j u i \leqslant j^{\prime}$. Тогда $\check{f}_{i j}(\lambda) / \delta_{j}^{-}(\lambda) \neq 0$ для всех $\lambda \in \mathfrak{h}^{*}$.

ДоКАЗАТЕЛЬСтво. Для $\mathfrak{g}=\mathfrak{s p}(2 n)$ имеем $\mathrm{s}=n$ и $\delta_{j}^{-}=1$. В силу леммы 4.2 достаточно проверить случай $i \leqslant n, n^{\prime} \leqslant j$. Вычисляя проекцию по модулю $J_{\mathrm{s}}$, получаем факторизацию $\check{f}_{i j}=\check{f}_{i n} \check{f}_{n n^{\prime}} \check{f}_{n^{\prime} j} y_{n^{\prime}}$. Она отлична от нуля, поскольку левый и правый множители в произведении не равны нулю в силу следствия 4.2 и соотношения $f_{n n^{\prime}}=[2]_{q} f_{n} \neq 0$. Заметим, что ограничение $i \leqslant j^{\prime}$ можно ослабить для симплектической алгебры $\mathfrak{g}$.

Случай ортогональной алгебры $\mathfrak{g}$ при $j<\mathrm{s}^{\prime}$ рассмотрен в следствии 4.2, поскольку в этой ситуации $\delta_{j}^{-}=1$.

Предположим теперь, что $\mathfrak{g}$ ортогональна и $s^{\prime} \leqslant j$ (подразумевая $i \leqslant s$ ). Проекция по модулю $J_{\mathrm{s}}$ дает равенство $\hat{f}_{i j} / \delta_{j}^{-}=\hat{f}_{i n}\left(\hat{f}_{\mathrm{ss}^{\prime}} / \delta_{j}^{-}\right) \hat{f}_{n^{\prime} j}$. Она отлична от нуля при условии $\hat{f}_{\mathrm{ss}^{\prime}} / \delta_{j}^{-} \neq 0$. Таким образом, мы нашли, что она равна $f_{n}^{2}$ для нечетных $N$ и $f_{n-1} f_{n}$ для четных $N$. Теорема доказана.

Доказанная теорема описывает регуляризацию $\hat{z}_{-\alpha_{j}}=\hat{f}_{1 j}$ отрицательных генераторов Микельсона для $\alpha=\varepsilon_{1}-\varepsilon_{j} \in \mathrm{R}^{+}$.

ПримеР 4.1. Проиллюстрируем теорему 4.1 на примере $\check{f}_{15}$ для $\mathfrak{g}=\mathfrak{s o}(6)$. Алгебра $\mathcal{A}_{5}$ порождается набором $y_{1}, \ldots, y_{4}$ при условии $y_{3} y_{4}=y_{2}$. Тогда

$$
d_{5}^{-}=\bar{A}_{2}=\frac{y_{2}-1}{q-\bar{q}} .
$$

При $f_{34}=0$ для $\hat{f}_{15}$ можно написать формулу

$$
\hat{f}_{15}=f_{15} A_{1}+f_{13} f_{35} A_{1,3}+f_{14} f_{45} A_{1,4}+f_{12} f_{25} A_{1,2}+f_{12} f_{23} f_{35} A_{1,2,3}+f_{12} f_{24} f_{45} A_{1,2,4} .
$$

Элементы $f_{1} f_{2} f_{3}, f_{13} f_{3}, f_{14} f_{2}$ и $f_{15}$ образуют базис в подпространстве веса $\varepsilon_{5}-\varepsilon_{1}$ в подалгебре $U_{q}\left(\mathfrak{g}_{-}\right)$. При $f_{12}=f_{1}, f_{23}=f_{45}=f_{2}, f_{24}=f_{35}=f_{3}$ перепишем соотношение $\check{f}_{15}=\hat{f}_{15} \bar{A}_{1} \ldots \bar{A}_{4}$ в виде

$$
\check{f}_{15}=f_{15} \bar{A}_{2} \bar{A}_{3} \bar{A}_{4}+f_{13} f_{3} \bar{A}_{4} \bar{A}_{2}+f_{14} f_{2} \bar{A}_{3} \bar{A}_{2}+f_{1} f_{2} f_{3}\left(q-\bar{q}+A_{3}+A_{4}\right) \bar{A}_{3} \bar{A}_{4} .
$$

Учитывая, что

$$
\left(q-\bar{q}+A_{3}+A_{4}\right) \bar{A}_{3} \bar{A}_{4}=\frac{y_{3} y_{4}-1}{q-\bar{q}}=\bar{A}_{2},
$$


получаем регуляризацию

$$
\check{f}_{15} / \delta_{5}^{-}=f_{15} \frac{y_{3}-1}{q-\bar{q}} \frac{y_{4}-1}{q-\bar{q}}+f_{13} f_{3} \frac{y_{4}-1}{q-\bar{q}}+f_{14} f_{2} \frac{y_{3}-1}{q-\bar{q}}+f_{1} f_{2} f_{3},
$$

которая никогда не обращается в нуль.

\section{5. РЕГУЛЯРИЗАЦИЯ \\ ПОЛОЖИТЕЛЬНЫХ ГЕНЕРАТОРОВ МИКЕЛЬСОНА}

В этом разделе мы регуляризуем генераторы Микельсона с положительными весами. Соответствие $f_{\alpha} \rightarrow e_{\alpha}$ продолжается до изоморфизма антиалгебр $\omega: U_{q}\left(\mathfrak{g}_{-}\right) \rightarrow$ $U_{q}\left(\mathfrak{g}_{+}\right)$. Обозначим $e_{j i}=\omega\left(f_{i j}\right) \in U_{q}\left(\mathfrak{g}_{+}\right)$.

Зафиксируем $j>1$. Для данной пары $j \prec l \leqslant N$ определим

$$
D_{l}^{j}=\frac{q^{\eta_{l 1}-\eta_{j 1}}}{\left[\eta_{j 1}-\eta_{l 1}\right]_{q}} \in \widehat{U}_{q}(\mathfrak{h})
$$

Положим $D_{\vec{m}}^{j}=D_{m_{1}}^{j} \ldots D_{m_{k}}^{j}$ для пути $\vec{m}=\left(m_{1}, \ldots, m_{k}\right)$ при $j \prec m$. Будем считать

$$
\hat{z}_{\alpha_{j}}=e_{j 1}+\sum_{j \prec \vec{m} \prec k \leqslant N}^{\varnothing} f_{j, \vec{m}, k} e_{k 1} D_{\vec{m}, k}^{j}(-1)^{|j-k|} q^{\eta_{j 1}-\eta_{k 1}} \in \widehat{U}_{q}(\mathfrak{g}), \quad j=2, \ldots, N,
$$

и $\check{z}_{\alpha_{j}}=\hat{z}_{\alpha_{j}} \prod_{j \prec l} \bar{D}_{l}^{j} \in U_{q}(\mathfrak{g})$. Элементы $\hat{z}_{\alpha_{j}}$ с $\alpha_{j}=\varepsilon_{1}-\varepsilon_{j} \in \mathrm{R}^{+}$образуют набор положительных генераторов Микельсона [10].

Введем автоморфизм $\tau$ антиалгебр $U_{q}\left(\mathfrak{g}_{-}\right)$, тождественный на генераторах Шевалле. Соотношения Серра подразумевают, что автоморфизм $\tau$ хорошо определен.

ЛЕмма 5.1. Для всех $i, j$ верно равенство $\tau\left(f_{i j}\right)=f_{j^{\prime} i^{\prime}}$.

ДоказАтельство. Рассмотрим явные выражения (2.3)-(2.8) для $f_{i j}$. Доказательство немедленно получается для общих линейных и нечетных ортогональных алгебр g. В других случаях наиболее трудная часть доказательства сводится к проверке равенства $\left[f_{j n}, f_{n i}\right]_{\bar{q}^{1+\delta_{i j^{\prime}}}}=\left[f_{j n^{\prime}}, f_{n^{\prime} i}\right]_{\bar{q}^{1+\delta_{i j^{\prime}}}}$ для $i<n, n^{\prime}<j, \mathfrak{g}=\mathfrak{s p}(2 n)$ и $\mathfrak{g}=\mathfrak{s o}(2 n)$. Можно осуществить проверку с помощью модифицированного тождества Якоби

$$
\left[x,[y, z]_{a}\right]_{b}=\left[[x, y]_{c}, z\right]_{a b / c}+c\left[y,[x, z]_{b / c}\right]_{a / c}
$$

для соответствующих значений $a, b, c \in \mathbb{C}$. Они выбираются так, чтобы уничтожить один из внутренних коммутаторов, используя соотношения в подалгебрах типа $A$ группы $U_{q}(\mathfrak{g})$. Лемма доказана.

Введем $\delta_{j}^{-} \in \mathcal{A}_{j}$ следующим образом. Положим $\delta_{j}^{-}=1$ для $j<\mathrm{s}^{\prime}$, a для $j \geqslant \mathrm{~s}^{\prime}$ будем считать $\delta_{j}^{-}=\left(y_{j^{\prime}}-1\right)(q-\bar{q})=\bar{A}_{j^{\prime}}$, если $\mathfrak{g}=\mathfrak{s o}(2 n)$, и $\delta_{j}^{-}=\left(y_{j^{\prime}}^{1 / 2}+1\right)(q+1)$, если $\mathfrak{g}=\mathfrak{s o}(2 n+1)$.

Обозначим

$$
\begin{aligned}
& \delta_{j}^{+}=\frac{q^{2 \eta_{j 1}-2 \eta_{j^{\prime} 1}}-1}{q-\bar{q}} \quad \text { для } \quad \mathfrak{s o}(2 n), \\
& \delta_{j}^{+}=\frac{q q^{2 \eta_{j 1}-2 \eta_{* 1}}+1}{q+1} \quad \text { для } \quad \mathfrak{s o}(2 n+1),
\end{aligned}
$$


предполагая $j \leqslant s$. Во всех других случаях, включая симплектическую алгебру $\mathfrak{g}$, положим $\delta_{j}^{+}=1$.

Теорема 5.1. Для каждого $j=2, \ldots, N$ элемент $\check{z}_{\alpha_{j}}$ делится на $\delta_{j}^{+}$. Фактор $\check{z}_{\alpha_{j}}(\lambda) / \delta_{j}^{+}(\lambda)$ не обращается в нуль при всех $\lambda \in \mathfrak{h}^{*}$.

ДоКАЗАТЕЛьСТво. Можно предположить, что $\mathfrak{g}$ ортогональна и $s^{\prime} \leqslant j^{\prime}$. Для всех $\lambda \in \mathfrak{h}^{*}$ определим $\check{z}_{j k} \in U_{q}\left(\mathfrak{g}_{-}\right), k=j, \ldots, N$, полагая $\check{z}_{j j}=\prod_{j<l} \bar{D}_{l}^{j}$ и $\check{z}_{j k}=$


По теореме Пуанкаре-Биркгофа-Витта для $U_{q}(\mathfrak{g})$ равенство $\check{z}_{\alpha_{j}}(\lambda)=0$ имеет место тогда и только тогда, когда $\check{z}_{j k}(\lambda)=0$ для всех $k$.

Специализация $y_{k^{\prime}}=q^{2 \eta_{j 1}-\eta_{k 1}}, j \prec k$, переводит $\delta_{j^{\prime}}^{-}$в $\delta_{j}^{+}$. Можно проверить, что алгебра, порожденная набором $\left\{y_{l}\right\}_{l<j^{\prime}}$, изоморфна алгебре $\mathcal{A}_{j^{\prime}}$. Действительно, $y_{k} y_{k^{\prime}}=y_{j}$ для всех $k \neq *$ и $q^{2} y_{*}^{2}=y_{j}$ (при нечетных $\left.N\right)$.

Антиавтоморфизм $\tau$ переводит $\check{z}_{j k}$ в $\check{f}_{k^{\prime} j^{\prime}}\left(\prod_{l} \bar{A}_{l}\right)$, где произведение берется по тем $l<j^{\prime}$, для которых верно неравенство $k^{\prime} \npreceq l$; в него входят все $\bar{A}_{l}$ с $k^{\prime}<l$. По лемме $3.3 \check{f}_{k^{\prime} j^{\prime}}$ делится на $\delta_{j^{\prime}}^{-}$, если $k^{\prime} \leqslant j$, в противном случае $\prod_{l} \bar{A}_{l}$ содержит $\bar{A}_{j}$, которая делится на $\delta_{j^{\prime}}^{-}$. Это доказывает, что $\check{z}_{\alpha_{j}}$ делится на $\delta_{j}^{+}$. Наконец, элемент $\check{f}_{1 j^{\prime}} / \delta_{j^{\prime}}^{-}$ является $\tau$-образом $\check{z}_{j N} / \delta_{j}^{+}$с точностью до множителя $(-1)^{|j-k|} y_{k^{\prime}} \neq 0$. Поскольку $\check{f}_{1 j^{\prime}} / \delta_{j^{\prime}}^{-}$не обращается в нуль для всех весов, $\check{z}_{\alpha_{j}} / \delta_{j}^{+} \neq 0$ тоже для всех весов. Теорема доказана.

\section{6. ПРИМЕНЕНИЕ РЕГУЛЯРИЗАЦИИ: РАЗЛОЖЕНИЕ $V \otimes M_{\lambda}$}

Определим возрастающую последовательность подмодулей $W_{j}=\sum_{i=1}^{j} M_{i} \subset V \otimes$ $M_{\lambda}$ для всех $j=1, \ldots, N$. Лемма 3.1 подразумевает, что $W_{j} \subset V_{j}$. Применим регуляризационный анализ, чтобы ответить на вопрос, в каком случае $V \otimes M_{\lambda}$ является прямой суммой $M_{j}$. Ясно, что это утверждение справедливо, если собственные числа $x_{j}$ оператора $\mathcal{Q}$ попарно различны. Однако обратное не верно.

ПрЕДЛОЖЕНИЕ 6.1. Следующие утверждения эквивалентны для всех $j \in[1, N]$ :

1) $V_{j}=W_{j}$

2) $V_{i}=W_{i}$ для всех $i \leqslant j$,

3) проекция $\wp_{i}: M_{i} \rightarrow V_{i} / V_{i-1}$ является изоморфизмом для всех $i \leqslant j$,

4) $W_{j}=\oplus_{i=1}^{j} M_{i}$.

ДоКАЗАТЕЛЬСтво. Поскольку все $M_{i}$ и $V_{i} / V_{i-1}$ являются модулями Верма одинакового старшего веса, третье утверждение эквивалентно тому, что $\wp_{i}$ сюръективно или инъективно. То, что первое утверждение вытекает из второго, доказывается тривиально. При $W_{1}=V_{1}$ предположим, что второе утверждение выполнено, и пусть значение $k>1$ является наименьшим среди таких, что $W_{k} \neq V_{k}$. Тогда $V_{k}$ и $W_{k}$ имеют различные кратности веса $\lambda+\varepsilon_{k}$, поэтому первое утверждение вытекает из второго.

Допустив справедливость второго утверждения, получаем, что все $\wp_{i}$ сюръективны, следовательно, справедливо третье утверждение. Наоборот, третье утверждение означает, что все отображения $W_{i} \rightarrow V_{i} / V_{i-1}$ сюръективны. Поскольку $W_{1}=V_{1}$, докажем второе утверждение методом индукции по $i$. 
Если $\wp_{i}$ инъективны, то $M_{i} \cap W_{i-1} \subset M_{i} \cap V_{i-1}=\{0\}$, т. е. третье утверждение влечет четвертое. Наконец, предположим справедливость четвертого утверждения и докажем справедливость третьего по индукции: из эквивалентности третьего и второго утверждений для $i<j$ следует, что отображение $\wp_{j}$ инъективно и поэтому является изоморфизмом. Предложение доказано.

Пусть $u_{j}=\check{F}_{j} / d_{j}^{-} \in V \otimes M_{\lambda}$ - регуляризованный сингулярный вектор, и введем $C_{j}(\lambda) \in \mathbb{C}$ с помощью равенства $\wp_{j}\left(u_{j}\right)=C_{j}(\lambda) v_{\lambda, j}$.

СлеДСТВИЕ 6.1. Модуль $V \otimes M_{\lambda}$ является прямой суммой $\oplus_{j=1}^{N} M_{j}$ тогда и только тогда, когда $C_{j}(\lambda) \neq 0$.

ДокАЗАТЕЛьство. Поскольку $u_{j}$ не обращается в нуль для всех $\lambda, C_{j}(\lambda)=0$ тогда и только тогда, когда $\wp_{j}$ является изоморфизмом. Следствие доказано.

Обозначим $\phi_{i j}=\left(q^{2 \xi_{i j}}-1\right)(q-\bar{q})$ при $i<j$, за исключением $i=j^{\prime}$ для $\mathfrak{g}=\mathfrak{s o}(N)$, а в этом случае положим $\phi_{j^{\prime} j}=1$ для четных $N$ и $\varphi_{j^{\prime} j}=\left(q^{\xi_{j^{\prime} j}}-1\right)(q-\bar{q})$ для нечетных $N$. Тогда $C_{j} \simeq \prod_{i=1}^{j-1} \phi_{i j}$ с точностью до числового множителя, который никогда не обращается в нуль. Вспомним, что $q^{2 \xi_{i j}}=x_{i} x_{j}^{-1}$, где $x_{i}$ являются собственными числами оператора $\mathcal{Q}$. Если все они различны, тогда очевидно, что $V \otimes M_{\lambda}=\oplus_{j=1}^{N} M_{j}$. Следовательно, обратное тоже верно для симплектической алгебры $\mathfrak{g}$. Для ортогональной алгебры $\mathfrak{g}$ спектр оператора $\mathcal{Q}$ не разделяет подмодули $M_{j}$ со всеми весами. Например, выберем вес $\lambda$ таким, что $q^{2\left(\lambda+\rho, \varepsilon_{j}\right)}=-1$. Тогда $x_{j} x_{j^{\prime}}^{-1}=q^{4\left(\lambda+\rho, \varepsilon_{j}\right)}=1$. Если все остальные $x_{l}$ попарно различны и отличны от $x_{j}$, то разложение в прямую сумму по-прежнему остается справедливым. Это явление делает возможным квантование граничных классов сопряженности Леви в работе [10].

\section{Список литературы}

[1] J. Mickelsson, "Step algebras of semi-simple subalgebras of Lie algebras", Rep. Math. Phys., 4:4 (1973), 307-318.

[2] Д. П. Желобенко, “Экстремальные проекторы и обобщенные алгебры Микельсона над редуктивными алгебрами Ли”, Изв. АН СССР. Сер. матем., 52:4 (1988), 758-773.

[3] D. P. Zhelobenko, " $S$-algebras and Verma modules over reductive Lie algebras", Док $\Omega$. АН CCCP, 273:4 (1983), 785-788.

[4] P. Kekäläinen, "Step algebras of quantum algebras of type A, B and D", J. Phys. A: Math. Gen., 29:5 (1996), 1045-1053.

[5] S. Khoroshkin, O. Ogievetsky, "Mickelsson algebras and Zhelobenko operators", J. Algebra, 319:5 (2008), 2113-2165.

[6] Р. М. Ашерова, Ю. Ф. Смирнов, В. Н. Толстой, "Проекционные операторы для простых групп Ли", ТМФ, 8:2 (1971), 255-271.

[7] Р. М. Ашерова, Ю. Ф. Смирнов, В. Н. Толстой, "Проекционные операторы для простых групп Ли. ІІ. Общая схема построения понижающих операторов. Случай групп $S U(n)$ ", ТМФ, 15:1 (1973), 107-119.

[8] V. N. Tolstoy, "Projection operator method for quantum groups", Special Functions 2000: Current Perspective and Future Directions, v. 30 (Arizona State University, Tempe, AZ, May 29 - June 9, 2000), NATO Science Series II: Mathematics, Physics and Chemistry, eds. J. Bustoz, M. E. H. Ismail, S. K. Suslov, Kluwer Acad. Publ., Dordrecht, 2001, 457-488. 
[9] A. Mudrov, "R-matrix and inverse Shapovalov form", J. Math. Phys., 57:5 (2016), 051706, 10 pp., arXiv: 1412.3384.

[10] T. Ashton, A. Mudrov, "R-matrix and Mickelsson algebras for orthosymplectic quantum groups", J. Math. Phys., 56:8 (2015), 081701, 8 pp.

[11] J. G. Nagel, M. Moshinsky, "Operators that lower or raise the irreducible vector spaces of $U_{n-1}$ contained in an irreducible vector space of $U_{n}$ ", J. Math. Phys., 6:5 (1965), 682-694.

[12] P.-Y. Hou, "Orthonormal bases and infinitesimal operators of the irreducible representations of group $U_{n} "$, Sci. Sinica, 15 (1966), 763-772.

[13] A. I. Molev, "Gelfand-Tsetlin bases for classical Lie algebras", Handbook of Algebra, 4, ed. M. Hazewinkel, Elsevier/North-Holland, Amsterdam, 2006, 109-170.

[14] K. Styrkas, V. Tarasov, A. Varchenko, "How to regularize singular vectors and kill the dynamical Weyl group", Adv. Math., 185:1 (2004), 91-135.

[15] A. Mudrov, "Orthogonal basis for the Shapovalov form on $U_{q}(\mathfrak{s l}(n+1))$ ", Rev. Math. Phys., 27:2 (2015), 1550004, 23 pp.

[16] V. Drinfel'd, "Quantum groups", Proceedings of the International Congress of Mathematicians (ICM), v. 1 (Berkeley, CA, August 3-11, 1986), eds. A. M. Gleason, AMS, Providence, RI, 1987, 798-820.

[17] V. Chari, A. Pressley, A guide to quantum groups, Cambridge Univ. Press, Cambridge, 1995.

[18] T. Ashton, A. Mudrov, "Representations of quantum conjugacy classes of orthosymplectic groups", Вопросы квантовой теории поля и статистической физики. 23, Зап. научн. сем. ПОМИ, 433, ПОМИ, СПб., 2015, 20-40, arXiv: 1502.02392. 